\title{
Role of non-collective excitations in heavy-ion fusion reactions and quasi-elastic scattering around the Coulomb barrier
}

\author{
S. Yusa, ${ }^{1}$ K. Hagino, ${ }^{1}$ and N. Rowley ${ }^{2}$ \\ 1 Department of Physics, Tohoku University, Sendai 980-8578, Japan \\ ${ }^{2}$ Institut de Physique Nucléaire, UMR 8608, CNRS-IN2P3 et Université de Paris Sud, 91406 Orsay Cedex, France
}

\begin{abstract}
Despite the supposed simplicity of double-closed shell nuclei, conventional coupled-channels calculations, that include all of the known collective states of the target and projectile, give a poor fit to the fusion cross section for the ${ }^{16} \mathrm{O}+{ }^{208} \mathrm{~Pb}$ system. The discrepancies are highlighted through the experimental barrier distribution and logarithmic derivative, that are both well defined by the precise experimental fusion data available. In order to broaden our search for possible causes for this anomaly, we revisit this system and include in our calculations a large number of non-collective states of the target, whose spin, parity, excitation energy and deformation paramter are known from high-precision proton inelastic-scattering measurements. Although the new coupled-channels calculations modify the barrier distribution, the disagreemnt with experiment remains both for fusion and for quasi-elastic (QE) scattering. We find that the Q-value distributions for large-angle $\mathrm{QE}$ scattering become rapidly more important as the incident energy increases, reflecting the trend of the experimental data. The mass-number dependence of the non-collective excitations is discussed.
\end{abstract}

PACS numbers: 24.10.Eq,25.70.Bc,25.70.Jj,21.10.Pc,

\section{INTRODUCTION}

In heavy-ion reactions at energies close to the Coulomb barrier, couplings between the relative motion and the internal degrees of freedom in the colliding nuclei play a crucial role. A well known example is the enhancement of subbarrier fusion cross sections, compared to the predictions of a simple potential model, due to strong couplings to collective excitations [1, 2]. Coupled-channels analyses have been performed for various heavy-ion systems taking into account such coupling effects and have successfully accounted for experimental data for fusion reactions as well as quasi-elastic scattering at backward angles [1].

Conventionally, a few low-lying collective excitations, such as vibrational modes in spherical nuclei or rotational excitations in deformed nuclei, as well as fewnucleon transfer channels have been taken into account in the coupled-channels calculations. In the eigenchannel representation, channel-coupling effects lead to a distribution of potential barriers 3], and it has been well established that the barrier distribution can be directly extracted from experimental fusion and quasielastic cross sections. For fusion reactions, the barrier distribution is defined as the second derivative of the product of center-of-mass energy $E_{\mathrm{cm}}$ and fusion cross section $\sigma_{\text {fus }}$ with respect to $E_{\mathrm{cm}}$, that is, $d^{2}\left(E_{\mathrm{cm}} \sigma_{\text {fus }}\right) / d E_{\mathrm{cm}}^{2}$ [4, 5]. For quasi-elastic scattering, it is defined as the first derivative of the ratio of the backward quasi-elastic scattering cross section to the Rutherford cross section with respect to center-of-mass energy, that is $-d\left(\sigma_{\mathrm{qel}}(\theta=\pi) / \sigma_{\mathrm{R}}(\theta=\pi)\right) / d E_{\mathrm{cm}}[6$, 7]. The fusion and quasi-elastic barrier distributions have been found to behave in a similar way to each other, though the quasi-elastic barrier distribution tends to be more smeared [6, 8]. These quantities are known to be sensitive to the channel coupling effects [1, 2, 5]. They can also serve for the determination of deformation parameters [9].

Although the coupled-channels method appears successful for heavy-ion subbarrier fusion reactions, there is a long standing problem of the method that, in order to reproduce experimental fusion data, a significantly larger value of the surface diffuseness of the nuclear potential is required, compared to the value found from fitting the scattering process [10, 11]. Furthermore, some recently obtained experimental data cannot be accounted for by the conventional coupled-channels method. For example, fusion cross sections for several systems at deep subbarrier energies have turned out to be much smaller than the predictions of conventional coupled-channels calculations 12 15]. Another example is the quasi-elastic barrier distribution for the ${ }^{20} \mathrm{Ne}+{ }^{90,92} \mathrm{Zr}$ systems. The conventional coupled-channels analysis that takes into account only the collective excitations of the colliding nuclei fails to explain the difference in the experimental quasi-elastic barrier distributions of these two systems [16]. That is, although the experimental barrier distribution for the ${ }^{20} \mathrm{Ne}+{ }^{92} \mathrm{Zr}$ system is much more smeared than that for the ${ }^{20} \mathrm{Ne}+{ }^{90} \mathrm{Zr}$ system, the coupled-channels calculations yield similar barrier distributions for both systems due to the much larger deformation of the ${ }^{20} \mathrm{Ne}$ nucleus. One possible reason for the smearing may be the effect of transfer reactions [17]. However the total transfer cross sections for these systems have been found to be almost the same [16]. Therefore the difference between the barrier distributions has been conjectured to arise from non-collective excitations, that are not taken into account explicitly in the coupled-channels calculations.

In order to discuss how non-collective excitations affect low-energy heavy-ion reactions, in Ref. [18] we have solved schematic one-dimensional coupled-channels equations with a gaussian potential barrier. There are sev- 
eral ways to describe the non-collective degrees of freedom 19 24]. In Ref. 18], we employed the random matrix theory, that has been applied to deep inelastic collisions by Agassi et al. 20 23]. We have shown that, by including non-collective excitations, the barrier penetration probabilities are suppressed at energies above the barrier leading to a smeared barrier distribution.

In this paper, we carry out a similar analysis in three dimensions, using realistic spectra for the non-collective excitations. For this purpose, we choose the ${ }^{16} \mathrm{O}+{ }^{208} \mathrm{~Pb}$ system. In addition to the experimental data for subbarrier and deep subbarrier fusion reactions [25, 26], as well as quasi-elastic scattering for this system at energies near the Coulomb barrier [27 30], almost all of the excited states of ${ }^{208} \mathrm{~Pb}$ up to $7.5 \mathrm{MeV}$ have been identified (spin, parity, excitation energy and deformation parameter) from high-resolution proton inelastic scattering measurements [31, 32]. We will include these ${ }^{208} \mathrm{~Pb}$ excited states in our coupled-channels calculations and discuss the role of non-collective excitations in heavy-ion reactions around the Coulomb barrier.

Note that a satisfactory description of the fusion cross sections as well as the fusion barrier distribution has not yet been obtained for this system with the conventional coupled-channels calculations [25, 33]. That is, the height of the main peak in the barrier distribution is overestimated by the coupled-channels calculations (see also Ref. [34]). Another motivation to choose the ${ }^{16} \mathrm{O}+{ }^{208} \mathrm{~Pb}$ system in the present study is, therefore, to see whether the non-collective excitations improve the agreement of the coupled-channels calculation with the experimental data.

In addition to our calculation for the ${ }^{16} \mathrm{O}+{ }^{208} \mathrm{~Pb}$ system, we also study ${ }^{32} \mathrm{~S}+{ }^{208} \mathrm{~Pb}$ and ${ }^{40} \mathrm{Ca}+{ }^{208} \mathrm{~Pb}$ to investigate the dependence of the effect of the noncollective excitations on the mass number of the projectile.

The paper is organized as follows. In Sec. II, we explain the coupled-channels formalism and how to describe the couplings to the non-collective excitations. In Sec. III, we apply the coupled-channels formalism to the fusion and quasi-elastic scattering of the ${ }^{16} \mathrm{O}+{ }^{208} \mathrm{~Pb}$ system. We will discuss fusion and quasi-elastic cross sections, barrier distributions, as well as the energy dependence of the Q-value distributions for quasi-elastic scattering. We also investigate the fusion reaction for the ${ }^{32} \mathrm{~S}+{ }^{208} \mathrm{~Pb}$ and ${ }^{40} \mathrm{Ca}+{ }^{208} \mathrm{~Pb}$ systems and discuss the mass-number dependence of the non-collective effects. In Sec. IV, we summarize the paper.

\section{COUPLED-CHANNELS METHOD}

In order to take into account excitations of the colliding nuclei during the fusion and scattering processes, we assume the following Hamiltonian:

$$
H=-\frac{\hbar^{2}}{2 \mu} \nabla^{2}+V_{\text {rel }}(r)+H_{0}(\xi)+V_{\text {coup }}(\boldsymbol{r}, \xi),
$$

where $\boldsymbol{r}$ is the coordinate for the relative motion between the projectile and the target nuclei, and $\mu$ is the reduced mass. $H_{0}(\xi)$ is the intrinsic Hamiltonian, for which we consider vibrational excitations of the colliding nuclei, $\xi$ representing the internal degrees of freedom. $V_{\text {coup }}(\boldsymbol{r}, \xi)$ is the coupling Hamiltonian between the relative motion and the intrinsic degrees of freedom. $V_{\text {rel }}(r)$ is the optical potential for the relative motion, that is given as a sum of the Coulomb and nuclear potentials,

$$
\begin{aligned}
V_{\text {rel }}(r)=\frac{Z_{\mathrm{P}} Z_{\mathrm{T}} e^{2}}{r} & -\frac{V_{0}}{1+\exp \left[\left(r-R_{\mathrm{N}}\right) / a\right]} \\
& -i \frac{W_{0}}{1+\exp \left[\left(r-R_{\mathrm{W}}\right) / a_{\mathrm{W}}\right]} .
\end{aligned}
$$

Here, we have adopted the Woods-Saxon form for the nuclear potential.

The coupled-channels equations are obtained by expanding the total wave function in terms of the eigenfunctions of $H_{0}(\xi)$. This leads to

$$
\begin{aligned}
{\left[-\frac{\hbar^{2}}{2 \mu} \frac{d^{2}}{d r^{2}}+\frac{J(J+1) \hbar^{2}}{2 \mu r^{2}}+\right.} & \left.V_{\text {rel }}(r)+\epsilon_{n}-E\right] u_{n}^{J}(r) \\
& +\sum_{m} V_{n m}(r) u_{m}^{J}(r)=0,
\end{aligned}
$$

where, $\epsilon_{n}$ is the excitation energy for the $n$-th channel. In deriving these equations, we have employed the isocentrifugal approximation [35 44] and replaced the angular momentum for the relative motion by the total angular momentum, $J$. This approximation has been found to be valid for heavy-ion systems [39], and reduces considerably the dimensions of the coupled-channels problem.

We impose the following boundary conditions in solving the coupled-channels equations:

$$
u_{n}^{J}(r) \rightarrow H_{J}^{(-)}\left(k_{n} r\right) \delta_{n, 0}-\sqrt{\frac{k_{0}}{k_{n}}} S_{n}^{J} H_{J}^{(+)}\left(k_{n} r\right),
$$

for $r \rightarrow \infty$, together with regularity at the origin. Here, $k_{n}=\sqrt{2 \mu\left(E-\epsilon_{n}\right) / \hbar^{2}}$ is the wave number for the $n$-th channel, where $n=0$ represents the entrance channel. $S_{n}^{J}$ is the nuclear $S$-matrix, and $H_{J}^{(-)}(k r)$ and $H_{J}^{(+)}(k r)$ are the incoming and the outgoing Coulomb wave functions, respectively. Using the $S$-matrix, the fusion cross sections are calculated as

$$
\sigma_{\text {fus }}(E)=\frac{\pi}{k_{0}^{2}} \sum_{J}(2 J+1)\left(1-\left|\sum_{n} S_{n}^{J}\right|^{2}\right) .
$$

On the other hand, the differential cross sections for the channel $n$ are given by

$$
\frac{d \sigma_{n}}{d \Omega}=\frac{k_{n}}{k_{0}}\left|f_{n}(\theta)\right|,
$$

with

$$
\begin{aligned}
f_{n}(\theta)= & \frac{1}{2 i \sqrt{k_{0} k_{n}}} \sum_{J} e^{i\left[\sigma_{J}(E)+\sigma_{J}\left(E-\epsilon_{n}\right)\right]} \\
& \times(2 J+1) P_{J}(\cos \theta)\left(S_{n}^{J}-\delta_{n, 0}\right) \\
& +f_{\mathrm{C}}(\theta) \delta_{n, 0}
\end{aligned}
$$


where $\sigma_{J}(E)$ and $f_{\mathrm{C}}(\theta)$ are the Coulomb phase shift and the Coulomb scattering amplitude, respectively. The quasi-elastic scattering cross sections are calculated according to

$$
\sigma_{\mathrm{qel}}(E, \theta)=\sum_{n} \frac{d \sigma_{n}}{d \Omega}(E, \theta)
$$

\section{RESULTS}

Let us now numerically solve the coupled-channels equations for the ${ }^{16} \mathrm{O}+{ }^{208} \mathrm{~Pb}$ system. For the coupling to the collective excitations, we take into account the vibrational $3^{-}$state at $2.615 \mathrm{MeV}, 5^{-}$state at $3.198 \mathrm{MeV}$, and $2^{+}$state at $4.085 \mathrm{MeV}$ in ${ }^{208} \mathrm{~Pb}$ as well as the $3^{-}$ state at $6.13 \mathrm{MeV}$ in ${ }^{16} \mathrm{O}$. The deformation parameters are estimated from the measured electromagnetic transition probabilities, that is, $\beta_{3}\left({ }^{208} \mathrm{~Pb}\right)=0.122, \beta_{5}\left({ }^{208} \mathrm{~Pb}\right)$ $=0.058, \beta_{2}\left({ }^{208} \mathrm{~Pb}\right)=0.058, \beta_{3}\left({ }^{16} \mathrm{O}\right)=0.733$, together with a radius parameter of $r_{0}=1.2 \mathrm{fm}$. In addition to these collective vibrational states, we also include 70 noncollective states in ${ }^{208} \mathrm{~Pb}$ below $7.382 \mathrm{MeV}$, whose excitation energies, multipolarities, and deformation parameters are taken from the high-resolution proton inelastic scattering measurements in Ref. 31]. We take into account the mutual excitations of the ${ }^{208} \mathrm{~Pb}$ and the ${ }^{16} \mathrm{O}$ nuclei.

For the nuclear potential, we use the same geometry as that in Ref. [29], where the parameters were obtained by fitting the coupled-channels calculations to the experimental quasi-elastic scattering cross sections. This potential has a surface diffuseness parameter of $a=$ $0.671 \mathrm{fm}$. Since our calculation takes into account the $3^{-}$ state in ${ }^{16} \mathrm{O}$, that was not included in Ref. [29], we modify the potential depth from $853 \mathrm{MeV}$ to $550 \mathrm{MeV}$ in order to compensate the adiabatic potential renormalization [45]. For the form factors of the non-collective couplings, for simplicity we take the same geometry as that for the collective couplings. For the non-collective excitations, we include only the couplings between the ground state and the non-collective states, and neglect the couplings among the non-collective excitations and the couplings between the collective and the non-collective states.

\section{A. Single phonon calculations}

We first show the results for the calculation that does not take into account double octupole phonon states in the ${ }^{208} \mathrm{~Pb}$. In this case, the number of channels amounts to 146 in the isocentrifugal approximation.

Figures 1(a) and 1(b) show the fusion cross sections thus obtained. They are plotted both on a linear scale (Fig. 1(a)) and on a logarithmic scale (Fig. 1(b)). The corresponding barrier distributions, $D_{\text {fus }}=d^{2}\left(E \sigma_{\text {fus }}\right) / d E^{2}$, are plotted in Fig. 1( $(\mathrm{c})$. The experimental data are taken from Refs. [25, 26]. The

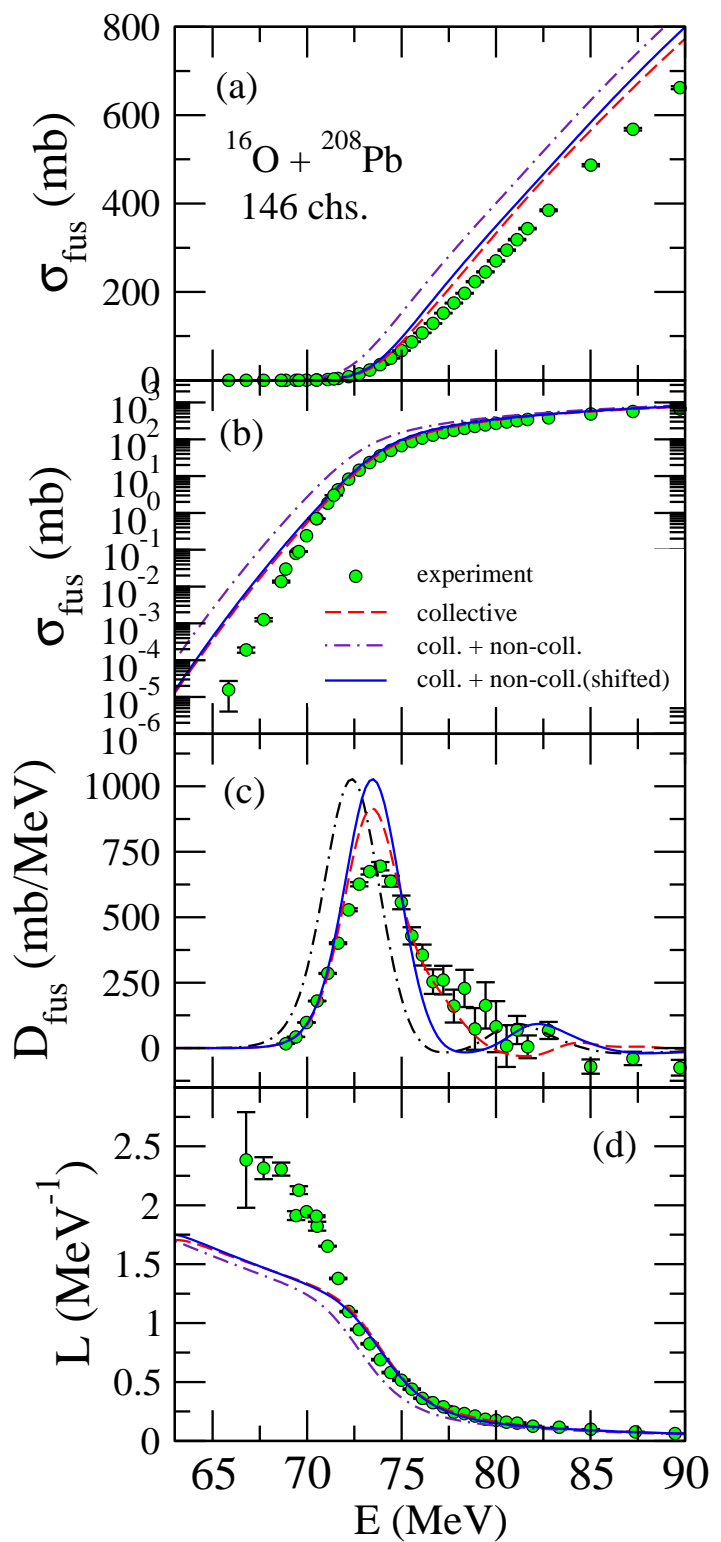

FIG. 1: (Color online) The fusion cross sections (Fig. 1(a) and $1(\mathrm{~b}))$, the fusion barrier distribution, $D_{\text {fus }}=$ $d^{2}\left(E \sigma_{\text {fus }}\right) / d E^{2},($ Fig. 1(c)), and the logarithmic slope, $L(E)=$ $d\left[\ln \left(E \sigma_{\text {fus }}\right)\right] / d E$, (Fig. $\left.1(\mathrm{~d})\right)$, for the ${ }^{16} \mathrm{O}+{ }^{208} \mathrm{~Pb}$ reaction. The fusion cross sections are plotted both on linear and logarithmic scales in Figs. 1(a) and 1(b), respectively. The dashed lines are obtained by taking into account only the collective excitations of ${ }^{16} \mathrm{O}$ and ${ }^{208} \mathrm{~Pb}$, while the dot-dashed lines take into account the non-collective excitations of ${ }^{208} \mathrm{~Pb}$ in addition to the collective excitations. The solid lines are the same as the dot-dashed lines, but shifted in energy. The experimental data are taken from Refs. 25, 26].

dashed lines are obtained by taking into account only the collective excitations of ${ }^{208} \mathrm{~Pb}$ and ${ }^{16} \mathrm{O}$, while the dot-dashed lines take into account also the non-collective excitations of ${ }^{208} \mathrm{~Pb}$. One immediately sees that the main peak in the barrier distribution is shifted in en- 
ergy due to the non-collective excitations towards low energy and consequently the fusion cross sections are enhanced. This can be understood in terms of the adiabatic potential renormalization because the excitation energies for the non-collective excitations are relatively large. One can also see that the non-collective excitations do not alter much the energy dependence of the fusion cross sections, as can be seen more clearly by shifting the dot-dashed lines in energy as shown in Fig. 1 by the solid lines. As a consequence, the non-collective excitations hardly modify the behavior of the logarithmic slope, $L(E)=d\left[\ln \left(E \sigma_{\text {fus }}\right)\right] / d E$ (see Fig. [1(d)). That is, the calculations with only the collective excitations do not account for the observed large logarithmic slope at deep subbarrier energies. This remains the same even if the non-collective excitations are taken into account. This indicates that the deep sub-barrier hindrance of fusion cross sections cannot be explained simply with the non-collective excitations in each of the colliding nuclei; some other mechanism, such as non-collective excitations of the one-body system after the touching of the colliding nuclei, has to be considered [34].

As mentioned in Sec. I, it is known that the calculation with only collective excitations does not reproduce well the experimental barrier distribution for this system [25]. That is, the coupled-channels calculation yields too high a main peak in the barrier distribution. We find that the non-collective excitations are not helpful in this respect, as shown in Fig. 1(c). The non-collective excitations rather smear the barrier distribution at energies around $78 \mathrm{MeV}$ [18], and the agreement is somewhat worsened. Clearly, one needs other mechanisms in order to reproduce the experimental barrier distribution for this system. In this connection, in the next subsection, we will investigate the effect of double octupole phonon excitations in ${ }^{208} \mathrm{~Pb}$.

Figure 2 shows the quasi-elastic scattering cross section and the quasi-elastic barrier distribution, $D_{\mathrm{qel}}(E)=$ $d\left[\sigma_{\mathrm{qel}} / \sigma_{\mathrm{R}}\right] / d E$ at $\theta_{\mathrm{cm}}=170^{\circ} . E_{\mathrm{eff}}$ is the effective energy defined by [6, 7]

$$
E_{\mathrm{eff}}=2 E \frac{\sin (\theta / 2)}{1+\sin (\theta / 2)}
$$

which takes into account the centrifugal energy for the Rutherford trajectory. The meaning of each line is the same as in Fig. 1. The solid lines are shifted in energy with the same amount as in the fusion calculation. The experimental data are taken from Ref. [28].

One can observe that the change in the barrier distribution due to the non-collective excitations is similar to the fusion calculation. That is, the main effect of the noncollective excitations is the barrier renormalization without changing the shape of the distribution, although they smear the barrier distributions at relatively higher energies. The agreement with the experimental data around $E_{\text {eff }}=75 \mathrm{MeV}$ is not improved by the non-collective excitations.

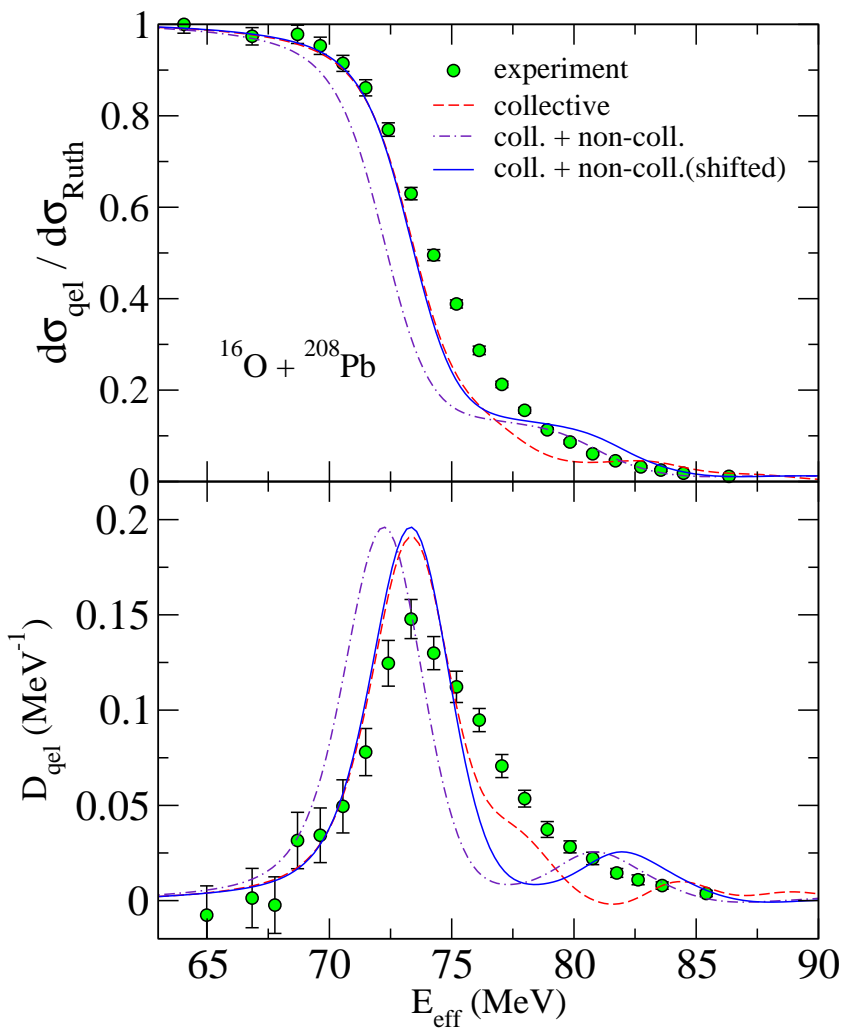

FIG. 2: (Color online) Quasi-elastic scattering cross sections (upper panel) and the quasi-elastic barrier distribution (lower panel) for the ${ }^{16} \mathrm{O}+{ }^{208} \mathrm{~Pb}$ system. The meaning of each line is the same as in Fig. 1]. Data are taken from Ref. 28].

\section{B. Double phonon calculation}

We next show the results for the calculations with the double octupole phonon excitations in ${ }^{208} \mathrm{~Pb}$. In this case, the number of channels included amounts to 148 . The double octupole phonon states in ${ }^{208} \mathrm{~Pb}$ have been experimentally investigated in Ref. [46 50] and candidates for the double phonon have been identified. In the present calculation we assume, for simplicity, that all four double octupole phonon states are degenerate with $E=5.23 \mathrm{MeV}$, that is, twice the energy of the singlephonon state.

In Figs. 3and 4, we show the calculations for the fusion reaction and quasi-elastic scattering, respectively. One sees that the double phonon excitations leads only to a minor improvement both for fusion and quasi-elastic scattering. The effects of the non-collective excitations are similar to those in the single-phonon case presented in the previous subsection. That is, the barrier distribution is smeared above the barrier while the shape of the lower peak is almost unchanged.

We have also investigated the role of anharmonicity of the octupole phonon excitations of ${ }^{208} \mathrm{~Pb}$ [51, 52], together with the non-collective excitations. We have found that the effect of anharmonicity is minor and again does 


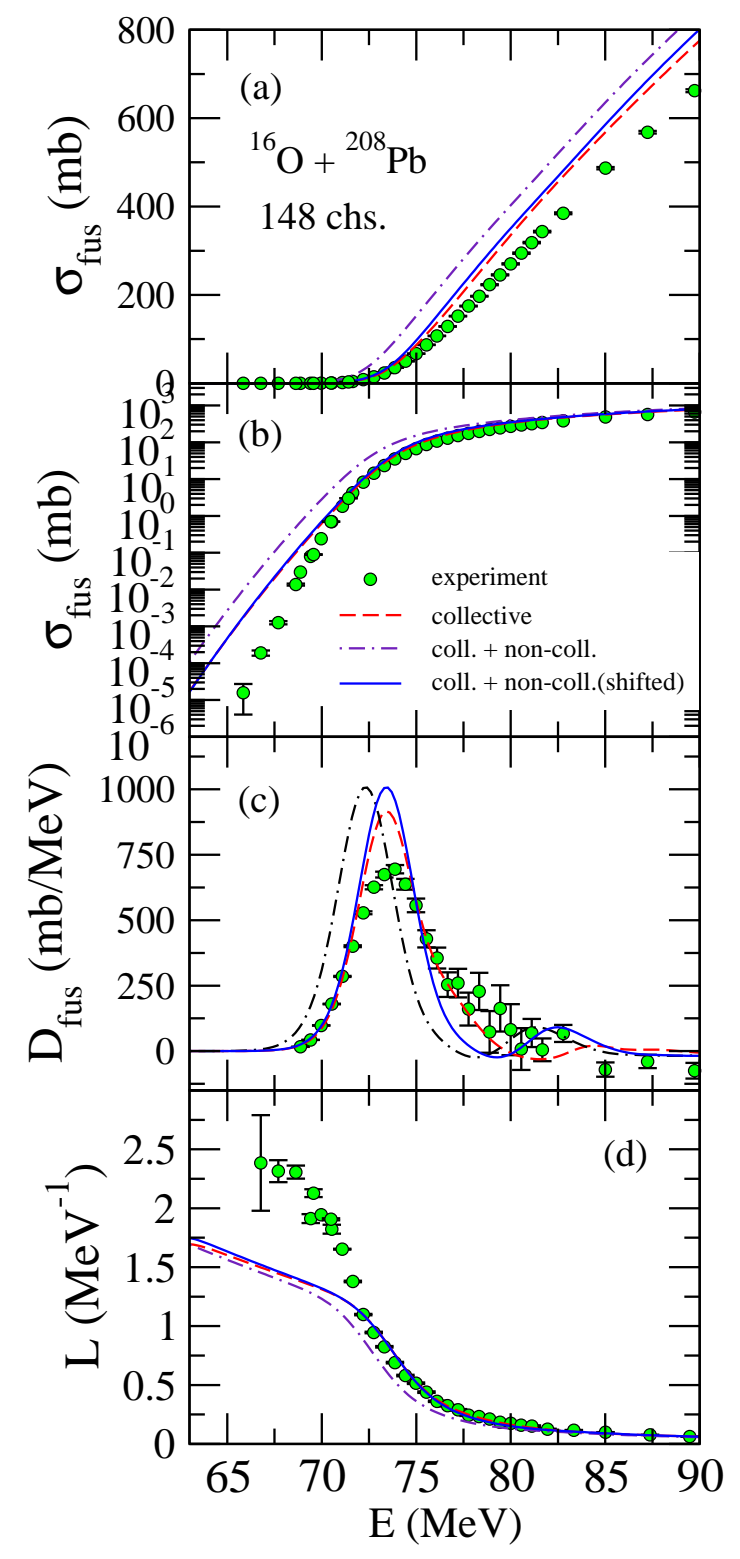

FIG. 3: (Color online) Same as Fig. 1 but with the double octupole phonon excitations.

not improve the agreement with the data.

\section{Q-value distribution}

Measurements of the Q-value distribution for backward-angle quasi-elastic scattering have been performed for this system [29, 30]. The experimental data indicate that the contribution from the non-collective excitations increases as the incident energy increases. A big advantage of our method is that the Q-value distribution can be computed easily because we explicitly take into account the non-collective excitations in our coupled-channels calculations.

Figure 5 shows the Q-value distributions at $\theta_{\mathrm{cm}}=170^{\circ}$

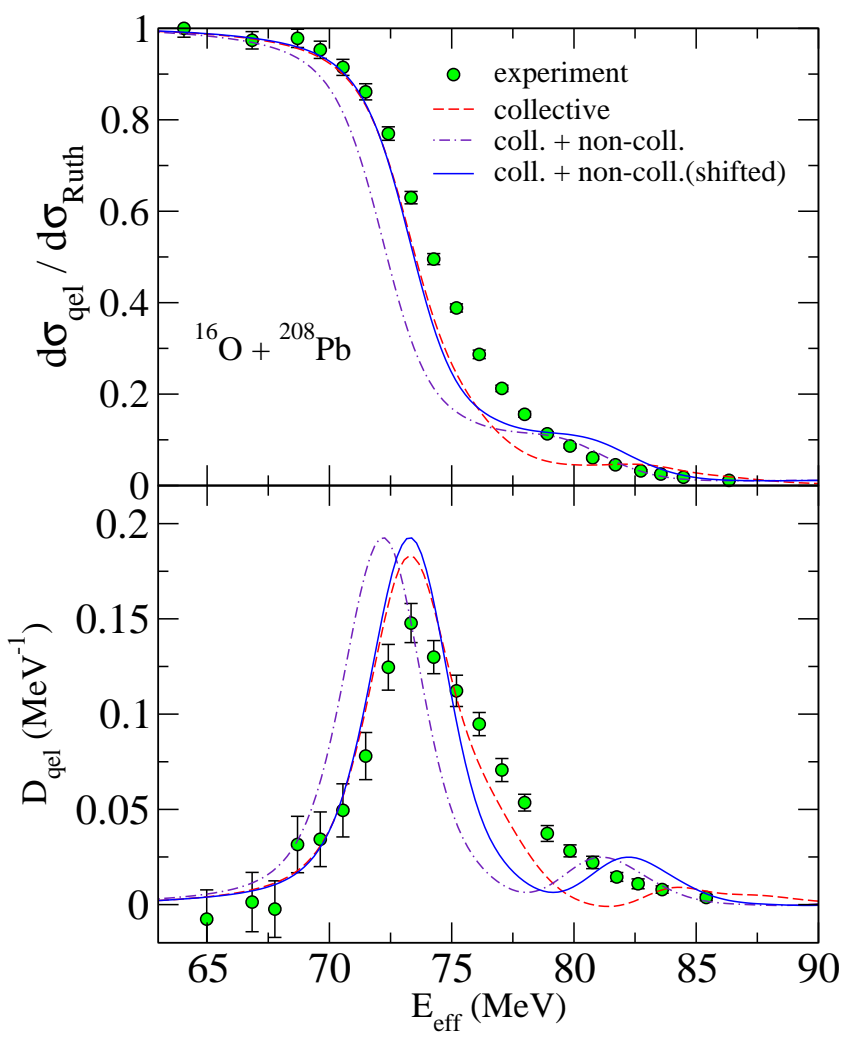

FIG. 4: (Color online) Same as Fig. 2, but with the double octupole phonon excitations.

at six different incident energies, corresponding to the double phonon calculations shown in Sec. III B. The spectra shown by the dashed lines correspond to the collective excitations while those by the solid lines correspond to the non-collective excitations. The envelope of the spectra is obtained by smearing with a gaussian function,

$$
F\left(E^{*}\right)=\sum_{n} \frac{d \sigma_{n}}{d \Omega} \frac{1}{\sqrt{2 \pi} \Delta} e^{-\frac{\left(E^{*}-\epsilon_{n}\right)^{2}}{2 \Delta^{2}}}
$$

with $\Delta=0.2 \mathrm{MeV}$.

Note that we include the non-collective states of ${ }^{208} \mathrm{~Pb}$ up to $7.382 \mathrm{MeV}$. Thus the spectra above this energy correspond to mutual excitations of the ${ }^{208} \mathrm{~Pb}$ and ${ }^{16} \mathrm{O}$ nuclei. One can see that, at the lowest incident energy shown in the figure, the contribution from the collective channels is dominant. With increasing energy, the contribution from the non-collective excitations becomes more and more important. This behaviour is consistent with the experimental Q-value distribution for this system [29, 30].

Note that this energy dependence is also related to how the non-collective excitations modify the energy dependence of the barrier distribution. Namely, at low energies where the contribution from the non-collective excitations is not important, a change in the barrier distribution is not observed. On the other hand, at higher ener- 


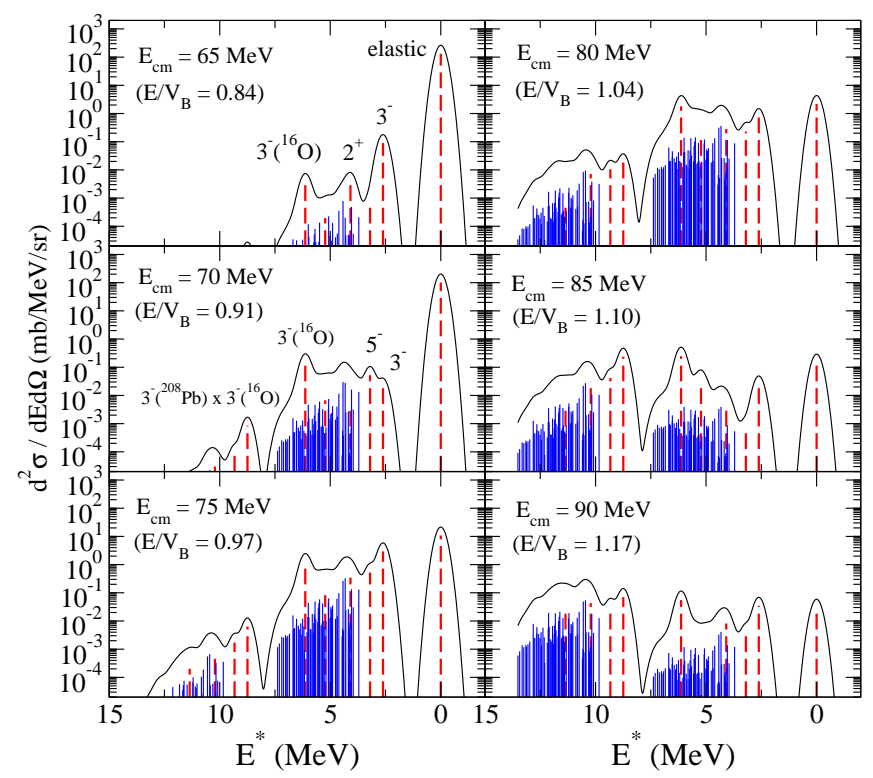

FIG. 5: (Color online)The Q-value spectra for the quasielastic scattering at $\theta_{\mathrm{cm}}=170^{\circ}$ for the ${ }^{16} \mathrm{O}+{ }^{208} \mathrm{~Pb}$ system for six different incident energies. The dashed peaks correspond to the collective excitations while the solid peaks correspond to the non-collective excitations. The solid line is obtained by smearing the peaks with a gaussian function.

gies where the contribution from the non-collective excitations is important, the barrier distribution is smeared due to the non-collective excitations.

\section{Mass-number dependence of the effect of non-collective excitations}

Finally, we investigate how the effect of non-collective excitations depends on the mass number of the projectile nucleus. For this purpose, we solve the coupled-channels equations for the ${ }^{32} \mathrm{~S}+{ }^{208} \mathrm{~Pb}$ and ${ }^{40} \mathrm{Ca}+{ }^{208} \mathrm{~Pb}$ systems. For the nuclear potential, we use the Akyüz-Winther potential [53]. We include the same excited states in the ${ }^{208} \mathrm{~Pb}$ nucleus as those in the calculation for the ${ }^{16} \mathrm{O}+$ ${ }^{208} \mathrm{~Pb}$ system discussed in the previous subsections.

We first discuss the ${ }^{32} \mathrm{~S}+{ }^{208} \mathrm{~Pb}$ reaction. For the excitations of ${ }^{32} \mathrm{~S}$, we take into account the quadrupole vibration up to double phonon states. The excitation energy and the deformation parameter are taken from Ref. [54]. Figure 6] shows the calculated fusion cross section and fusion barrier distribution. The meaning of each line is the same as in Fig. 1. The experimental data are taken from Ref. 28]. One can see that the effect of the non-collective excitations is qualitatively similar to that in the ${ }^{16} \mathrm{O}+{ }^{208} \mathrm{~Pb}$ reaction. That is, the barrier is shifted towards lower energy and the higher part of the barrier distribution is smeared. However, the smearing is stronger than that in the ${ }^{16} \mathrm{O}+{ }^{208} \mathrm{~Pb}$ system, because an effective coupling strength is in general approximately proportional to the charge product of the colliding nu-

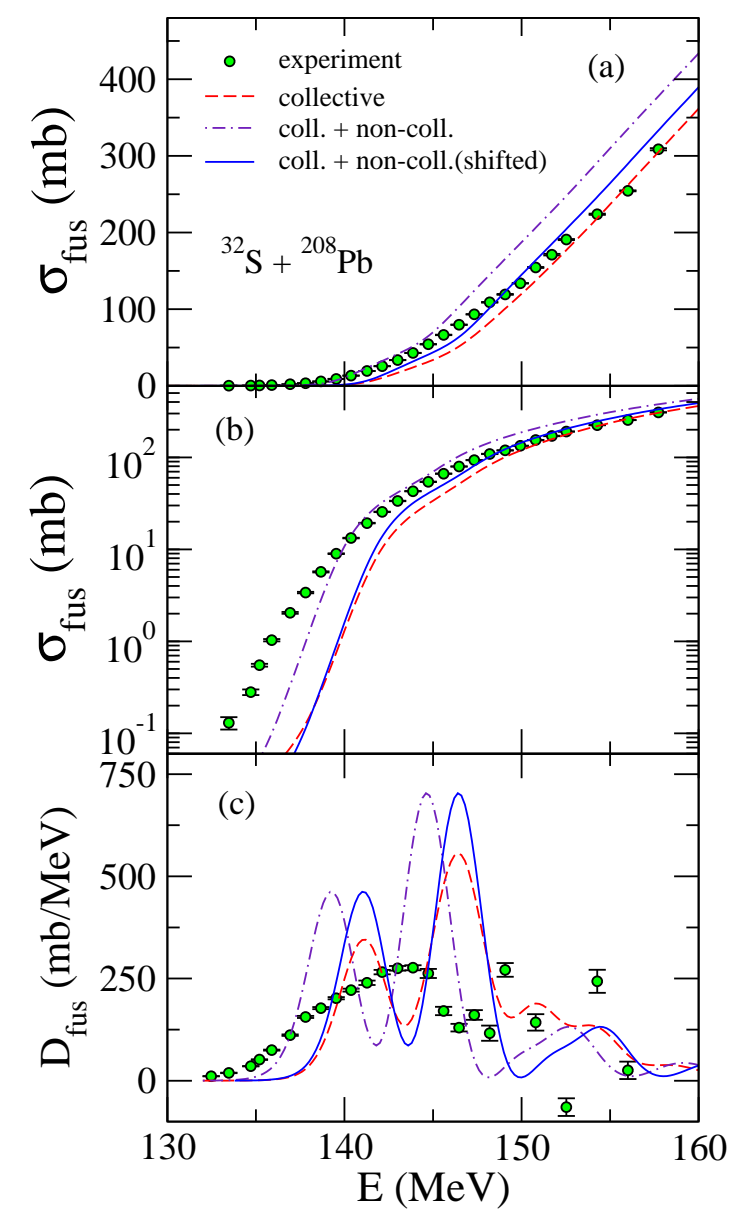

FIG. 6: (Color online) Fusion cross section and fusion barrier distribution for the ${ }^{32} \mathrm{~S}+{ }^{208} \mathrm{~Pb}$ system. The meaning of each line is the same as in Fig. 1] The experimental data are taken from Ref. [28].

clei [4], and thus the non-collective excitations are effectively stronger for heavier systems. One can also see that the two low-energy peaks in the barrier distribution are sharpened due to the non-collective excitations, while the separation between the peaks is not altered much. The calculations do not reproduce the experimental data, and this might be attributed to the role of transfer reactions.

Figure 7 shows the fusion cross section and the fusion barrier distribution for the ${ }^{40} \mathrm{Ca}+{ }^{208} \mathrm{~Pb}$ reaction. For this system, we assume that ${ }^{40} \mathrm{Ca}$ is inert and take into account only the excitations of ${ }^{208} \mathrm{~Pb}$. As the charge product is larger, the effect of the non-collective excitations is stronger than that in the ${ }^{16} \mathrm{O}+{ }^{208} \mathrm{~Pb}$ and ${ }^{32} \mathrm{~S}+$ ${ }^{208} \mathrm{~Pb}$ reactions. It smears the higher part of the barrier distribution while the lower main peak is sharpened.

As we have shown, while the effect of non-collective excitations is not large for the ${ }^{16} \mathrm{O}+{ }^{208} \mathrm{~Pb}$ system, the effect becomes increasingly important for heavier systems, such as ${ }^{40} \mathrm{Ca}+{ }^{208} \mathrm{~Pb}$. This suggests that the conventional coupled-channels approach, that neglects the non-collective excitations, is well justified for relatively 


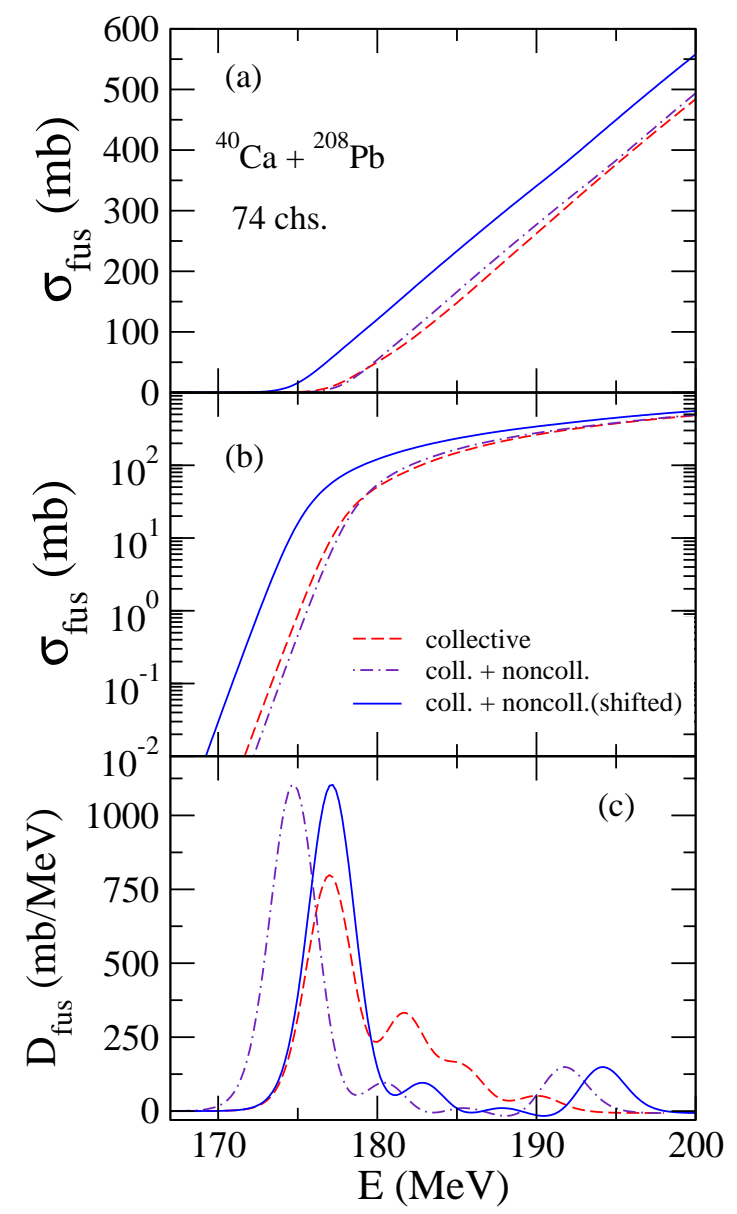

FIG. 7: (Color online) Fusion cross section and fusion barrier distribution for the ${ }^{40} \mathrm{Ca}+{ }^{208} \mathrm{~Pb}$ system. The meaning of each line is the same as in Fig. 1

light systems, but the non-collective excitations have to be included explicitly in coupled-channels calculations for heavy-systems, for example, those relavant to a synthesis of superheavy elements.

\section{SUMMARY}

We have solved the coupled-channels equations for fusion and quasi-elastic scattering for the ${ }^{16} \mathrm{O}+{ }^{208} \mathrm{~Pb}$ system, including both the collective and non-collective excitations of ${ }^{208} \mathrm{~Pb}$. Non-collective excitations are approximated by vibrational couplings, whose coupling strength and excitation energy are taken from the analysis of the high-resolution proton inelastic scattering experiment.

Our results show that the barrier distributions for the fusion reaction and the quasi-elastic scattering are changed in a similar manner due to the non-collective excitations at energies above the Coulomb barrier. The energy dependence of the cross sections, on the other hand, is not affected much by the non-collective excitations and the degree of agreement with the experimental barrier distributions remains the same. In order to improve the agreement, one would therefore have to consider another mechanism, such as a reduction of the excitation energy of the $3^{-}$state in ${ }^{208} \mathrm{~Pb}$ as suggested in Ref. [58].

The fusion calculations are also performed for the ${ }^{32} \mathrm{~S}$ $+{ }^{208} \mathrm{~Pb}$ and ${ }^{40} \mathrm{Ca}+{ }^{208} \mathrm{~Pb}$ systems in order to investigate the projectile mass-number dependence of the effect of the non-collective excitations. We have shown that the effect of the non-collective excitations becomes stronger as the mass number of the projectile nucleus increases.

For the ${ }^{32} \mathrm{~S}+{ }^{208} \mathrm{~Pb}$ system, the coupled-channels calculations with only the inelastic excitations of the colliding nuclei do not account for the experimental data. That is, the subbarrier fusion cross sections are significantly underestimated in this case and the experimental barrier distribution is much more smeared than that obtained by the coupled-channels calculation. The transfer process, as well as the non-collective excitations, should be taken into account for this system in order to improve the agreement with the data.

We also calculated the energy dependence of the Qvalue distribution for the ${ }^{16} \mathrm{O}+{ }^{208} \mathrm{~Pb}$ system and found that the contribution from the non-collective excitations becomes more and more important as the incident energy increases. This behaviour is consistent with the experimental Q-value distribution for the same system.

In this study, we have investigated systems involving the ${ }^{208} \mathrm{~Pb}$ nucleus because good information on its noncollective excitations is available. However, in general, such information is not necessarily available for other systems. For the ${ }^{90,92} \mathrm{Zr}$ nuclei, several proton inelastic scattering experiments have been performed [55 57]. However, the number of identified levels is not as large as for ${ }^{208} \mathrm{~Pb}$. Thus, we will have to use an approach different from this work, for example the random matrix theory [18], to describe the non-collective excitations in the ${ }^{20} \mathrm{Ne}+{ }^{90,92} \mathrm{Zr}$ system.

\section{Acknowledgments}

This work was supported by the Global COE Program "Weaving Science Web beyond Particle-Matter Hierarchy" at Tohoku University, and by the Japanese Ministry of Education, Culture, Sports, Science and Technology by Grant-in-Aid for Scientific Research under the program number (C) 22540262.
[1] M. Dasgupta, D.J. Hinde, N. Rowley, and A.M. Stefanini, Annu. Rev. Nucl. Part. Sci. 48, 401(1998).
[2] A. B. Balantekin and N. Takigawa, Rev. Mod. Phys. 70, $77(1998)$. 
[3] C.H. Dasso, S. Landowne, and A. Winther, Nucl. Phys. A405, 381 (1983); A407, 221 (1983).

[4] N. Rowley, G.R. Satchler and P.H. Stelson, Phys. Lett. B254 25, (1991).

[5] J.R. Leigh et al., Phys. Rev. C52, 3151 (1995).

[6] H. Timmers, J.R. Leigh, M. Dasgupta, D.J. Hinde, R.C. Lemmon, J.C. Mein, C.R. Morton, J.O. Newton, and N. Rowley, Nucl. Phys. A584, 190 (1995).

[7] K. Hagino and N. Rowley, Phys. Rev.C 69, 054610(2004).

[8] Muhammad Zamrun F. and K. Hagino, Phys. Rev. C77, 014606 (2008).

[9] J. R. Leigh, N. Rowley, R. C. Lemmon, D. J. Hinde, J. O. Newton, J. X. Wei, J. C. Mein, C. R. Morton, S. Kuyucak, and A. T. Kruppa, Phys. Rev. C47, R437(1993).

[10] A. Mukherjee, D. J. Hinde, M. Dasgupta, K. Hagino, J. O. Newton, and R. D. Butt, Phys. Rev. C75, 044608(2007).

[11] J.O. Newton et al., Phys. Lett. B586, 219 (2004); Phys. Rev. C70, 024605 (2004).

[12] C.L. Jiang et al., Phys. Rev. Lett. 89 (2002) 052701; Phys. Rev. C79 (2009) 044601, and references therein.

[13] C. L. Jiang, K. E. Rehm, R. V. F. Janssens, H. Esbensen, I. Ahmad, B. B. Back, P. Collon, C. N. Davids, J. P. Greene, D. J. Henderson, G. Mukherjee,*, R. C. Pardo, M. Paul, T. O. Pennington,, D. Seweryniak, S. Sinha, and Z. Zhou, Phys. Rev. Lett. 97, 012701(2004).

[14] M. Dasgupta et al., Phys. Rev. Lett. 99 (2007) 192701.

[15] A.M. Stefanini et al., Phys. Rev. C78 (2008) 044607; Phys. Lett. B679 (2009) 95.

[16] E. Piasecki, E. Świderski, W. Gawlikowicz, J. Jastrzebski, N. Keeley, M. Kisieliński, S. Kliczewski, A. Kordyasz, M. Kowalczyk, S. Khlebnikov, E. Koshchiy, E. Kozulin, T. Krogulski, T. Loktev, M. Mutterer, K. Piasecki, A. Piórkowska, K. Rusek, A. Staudt, M. Sillanpää, S. Smirnov, I. Strojek, G. Tiourin, W. H. Trzaska, A. Trzcińska, K. Hagino, and N. Rowley, Phys. Rev. C80, 054613 (2009).

[17] H. Timmers, L. Corradi, A.M. Stefanini, D. Ackermann, J.H. He, S. Beghini, G. Montagnoli, F. Scarlassara, G.F. Segato, N. Rowley, Phys. Lett. B399, 25 (1997).

[18] S. Yusa, K. Hagino, and N. Rowley, Phys. Rev. C82, 024606(2010).

[19] A. Diaz-Torres, Phys. Rev. C82, 054617(2010).

[20] D. Agassi, C.M. Ko, and H.A. Weidenmüller, Ann. Phys 107, 140(1977).

[21] C.M. Ko, D. Agassi, and H.A. Weidenmüller, Ann. Phys 117, 237(1979).

[22] D. Agassi, C.M. Ko, and H.A. Weidenmüller, Ann. Phys 117, 435 (1979).

[23] D. Agassi, C.M. Ko, and H.A. Weidenmüller, Phys. Rev. C 18, 223(1978).

[24] V.I. Zagrebaev, Ann. of Phys. (N.Y.) 197, 33 (1990).

[25] C. R. Morton, A. C. Berriman, M. Dasgupta, D. J. Hinde, and J. O. Newton, K. Hagino, and I. J. Thompson, Phys. Rev. C60, 044608(1999).

[26] M. Dasgupta, D. J. Hinde, A. Diaz-Torres, B. Bouriquet, Catherine I. Low, G. J. Milburn, and J. O. Newton, Phys. Rev. Lett.99, 192701(2007).

[27] H. Timmers et al., J. of Phys. G23, 1175 (1997).

[28] H. Timmers, Ph. D. thesis, Australian National University, 1996.

[29] M. Evers, M. Dasgupta, D.J. Hinde, L.R. Gasques, M.L. Brown, R. Rafiei, and R.G. Thomas, Phys. Rev. C78,
034614 (2008).

[30] C.J. Lin, H.M. Jia, H.Q. Zhang, F. Yang, X.X. Xu, F. Jia, Z.H. Liu, and K. Hagino, Phys. Rev. C79, 064603 (2009).

[31] W. T. Wagner, G. M. Crawley, G. R. Hammerstein, and H. McManus, /Phys. Rev. C12, 757(1975).

[32] M. B. Lewis, F. E. Bertrand, and C. B. Fulmer, Phys. Rev. C7, 1966(1973).

[33] H. Esbensen and S. Misicu, Phys. Rev. C76, 054609 (2007).

[34] T. Ichikawa, K. Hagino, and A. Iwamoto, Phys. Rev. Lett. 103, 202701 (2009).

[35] R. Lindsay and N. Rowley, J. Phys. G10, 805 (1984).

[36] M.A. Nagarajan, N. Rowley, and R. Lindsay, J. Phys. G12, 529 (1986).

[37] M.A. Nagarajan, A.B. Balantekin, and N. Takigawa, Phys. Rev. C34, 894 (1986).

[38] H. Esbensen, S. Landowne, and C. Price, Phys. Rev. C36, 1216 (1987); C36, 2359 (1987).

[39] O. Tanimura, Phys. Rev. C35, 1600 (1987); Z. Phys. A327, 413 (1987).

[40] N. Takigawa, F. Michel, A.B. Balantekin, and G. Reidemeister, Phys. Rev. C44, 477 (1991).

[41] N. Takigawa, Y. Alhassid, and A. B. Balantekin, Phys. Rev. C 45, 1850(1992).

[42] Y. Alhassid and H. Attias, Nucl. Phys. A577, 709 (1994).

[43] J. Gomez-Camacho, M.V. Andres, and M.A. Nagarajan, Nucl. Phys. A580, 156 (1994).

[44] K. Hagino, N. Takigawa, A. B. Balantekin, and J. R. Bennett, Phys. Rev. C52, 286(1995).

[45] N. Takigawa, K. Hagino, M. Abe, and A.B. Balantekin, Phys. Rev. C49, 2630 (1994).

[46] Minfang Yeh, P. E. Garrett, C. A. McGrath, S. W. Yates, and T. Belgya, Phys. Rev. Lett 76, 1208 (1996).

[47] K. Vetter et al., Phys. Rev. C 56, 2316 (1997).

[48] Minfang Yeh, M. Kadi, P. E. Garrett, C. A. McGrath, S. W. Yates, and T. Belgya, Phys. Rev. C 57 R2085 (1998).

[49] K. Vetter et al., Phys. Rev. C 58, R2631 (1998).

[50] B. D. Valnion, V. Yu. Ponomarev, Y. Eisermann, A. Gollwitzer, R. Hertenberger, A. Metz, P. Schiemenz, and G. Graw, Phys. Rev. C 63, 024318 (2001).

[51] K. Hagino, N. Takigawa, and S. Kuyucak, Phys. Rev. Lett. 79, 2943 (1997); K. Hagino, S. Kuyucak, and N. Takigawa, Phys. Rev. C57, 1349 (1998).

[52] Muhammad Zamrun F. and K. Hagino, Phys. Rev. C77, 014606 (2008).

[53] O. Akyüz and A. Winther, in Proc. of the Enrico Fermi School of Physics, 1979, Course on Nuclear Structure and Heavy-Ions Reactions, Editors R. A. Broglia, C. H. Dasso, and R. Ricci (North Holland, Amsterdam, 1981).

[54] S. Raman, C. W. Nestor Jr., and P. Tikkanen, At. Data Nucl. Data Tables 78, 1 (2001).

[55] M. M. Stautberg and J. J. Kraushaar, Phys. Rev. 151, 969(1966).

[56] J. K. Dickens, E. Eichler, and G. R. Satchler, Phys. Rev. 168, 1355(1968).

[57] F. Todd Baker, Alan Scott, M. A. Grimm, W. G. Love, V. Penumetcha, C. Glashausser, G. S. Adams and, G. Igo, G. W. Hoffmann, J. M. Moss, W. Swenson, B. E. Wood, Nucl. Phys. A393, 283(1983).

[58] N. Rowley and K. Hagino, Nucl. Phys. A834, 110c (2010). 